\title{
Cardiopulmonary Arrest and Extracorporeal Membrane Oxygenation: Case Report and Review
}

\author{
Alexander Robinson", Aniket S. Rali1,", Zubair Shah¹, Travis Abicht², Eric Hockstad ${ }^{1}$, Andrew Sauer ${ }^{1}$ \\ Departments of Internal Medicine, ${ }^{1}$ Cardiovascular Diseases and ${ }^{2}$ Cardiothoracic Surgery, University of Kansas Medical Center, Kansas City, Kansas, USA \\ *These authors contributed equally
}

\section{Abstract}

National trends suggest that less than one in four patients experiencing in-hospital cardiac arrest (IHCA) in the United States survive to discharge. This is especially relevant as the rates of IHCA are expected to rise in the years to come. Only a modest upward trend in survival to discharge among patients with IHCA over the past decade warrants evaluation of novel ideas to improve outcomes postcardiopulmonary resuscitation. One such idea is that the use of veno-arterial-extracorporeal membrane oxygenation (VA-ECMO) to augment standard advanced cardiac life support algorithm in patients with an identifiable and reversible cause of cardiac arrest would improve survival to discharge. Here, we present the case of a patient with refractory ventricular fibrillation arrest who was transitioned to VA-ECMO immediately following cardiac catheterization for an IHCA.

Keywords: Cardiopulmonary arrest, critical care cardiology, extracorporeal membrane oxygenation, extracorporeal membrane oxygenation-assisted cardiopulmonary resuscitation, intrahospital arrest

\section{INTRODUCTION}

In-hospital cardiac arrest (IHCA) affects approximately 200,000 hospitalized patients annually in the United States and this rate is expected to rise. ${ }^{[1]}$ Recent analysis of Get with the Guidelines-Resuscitation registry showed that between 2000 and 2014, acute resuscitation survival among patients with IHCA improved from $46.9 \%$ to $68.2 \%$. However, there was only a modest upward trend in survival to discharge among patients with IHCA during this period. ${ }^{[2]}$ Increasing incidence of IHCA and less than one in four patients surviving to discharge warrant evaluation of novel ideas to improve outcomes postcardiopulmonary resuscitation (CPR).

In refractory cardiac arrest, veno-arterial-extracorporeal membrane oxygenation (VA-ECMO)-assisted CPR (E-CPR) has been proposed for both IHCA and out-of-hospital cardiac arrest (OHCA). It is postulated that augmentation of standard advanced cardiac life support algorithm with the use of VA-ECMO in patients with an identifiable and reversible cause of cardiac arrest would improve survival to discharge. Review of the current medical literature yields mixed results about outcomes with the use of E-CPR, and hence, this has not become the standard of care yet. ${ }^{[3-5]}$ However, in the

\begin{tabular}{|l|l|}
\hline \multicolumn{3}{c|}{ Access this article online } \\
\hline Quick Response Code: & Website: \\
& www.ijccm.org \\
\hline
\end{tabular}

appropriate patient, implementation of E-CPR could prove lifesaving.

Here, we present a case of a patient with refractory ventricular fibrillation (V-Fib) arrest who was transitioned to VA-ECMO immediately following cardiac catheterization for an IHCA.

\section{Case Report}

Our patient is a 45 -year-old female who presented to the hospital with acute-onset left-sided chest pain. Her past medical history was significant for tobacco abuse and a miscarriage 1 month ago. Of note, the patient had had five normal pregnancies prior to this. Electrocardiogram performed in the emergency room (ER) was consistent with anterior ST-segment myocardial infarction [Figure 1]. Pertinent labs in the ER included a potassium level of $3.4 \mathrm{mmol} / \mathrm{L}$, troponin of $0.03 \mathrm{ng} / \mathrm{ml}$ that rose to $>70 \mathrm{ng} / \mathrm{ml}$ within $10 \mathrm{~h}$, serum creatinine of $0.81 \mathrm{mg} / \mathrm{dl}$, and negative

Address for correspondence: Dr. Aniket S. Rali, 3901 Rainbow Blvd, MS 3006, 1001 Eaton, Kansas City, KS 66160, USA. E-mail: arali@kumc.edu

This is an open access journal, and articles are distributed under the terms of the Creative Commons Attribution-NonCommercial-ShareAlike 4.0 License, which allows others to remix, tweak, and build upon the work non-commercially, as long as appropriate credit is given and the new creations are licensed under the identical terms.

For reprints contact: reprints@medknow.com

How to cite this article: Robinson A, Rali AS, Shah Z, Abicht T, Hockstad E, Sauer A. Cardiopulmonary arrest and extracorporeal membrane oxygenation: Case report and review. Indian J Crit Care Med 2018;22:544-6. 


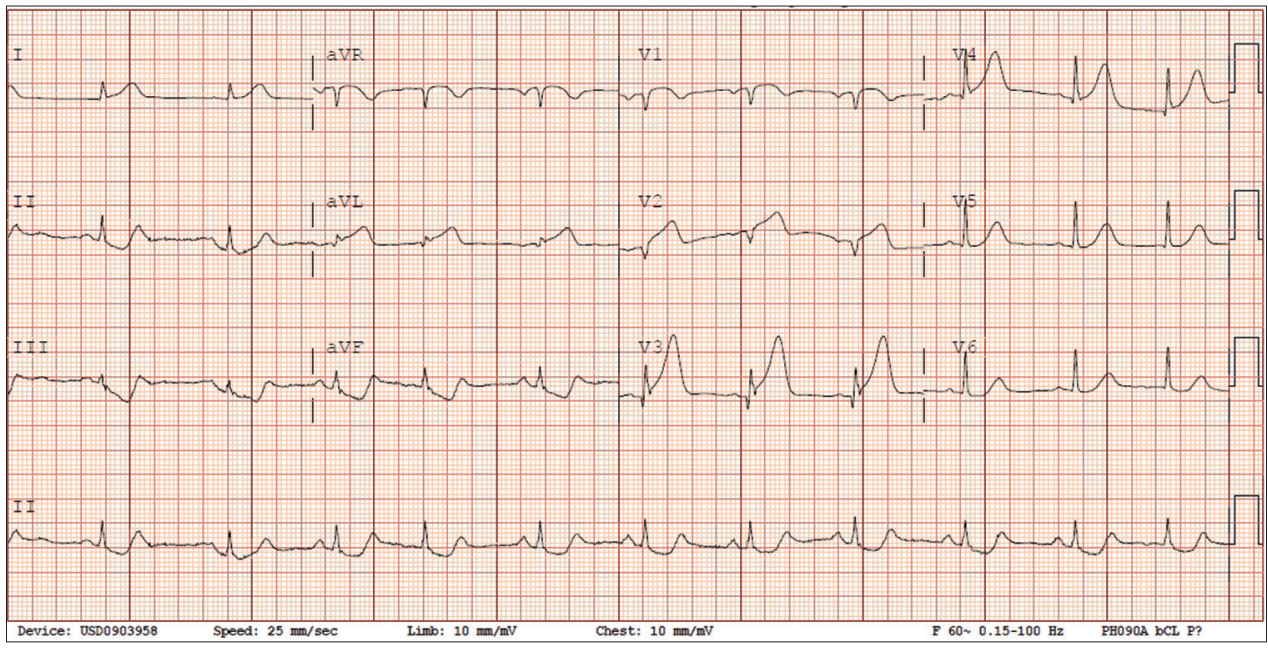

Figure 1: Presenting electrocardiogram consistent with anterior ST-segment elevation myocardial infarction

pregnancy test. Unfortunately, before the patient could be transferred to the cardiac catheterization lab, she went into V-Fib arrest. During the code, our cardiogenic shock team was activated for patient to be transitioned to VA-ECMO for mechanical circulatory support while the coronary angiography could be performed. After about $12 \mathrm{~min}$ of performing CPR, return of spontaneous circulation (ROSC) was achieved and the patient was emergently transferred to the catheterization lab. While in the lab, patient went back into V-Fib and had to be shocked out of it twice. At this point, intra-aortic balloon pump was placed. Coronary angiography showed an ostial left anterior descending artery occlusion [Figure 2] that had intervention performed with a drug-eluding stent [Figure 3]. Good angiographic results were obtained, but the patient continued to have multiple runs of sustained ventricular arrhythmias and hence the patient was placed on VA-ECMO while still in the catheterization lab. Subsequently, the patient was transferred to our Intensive Care Unit, remained hemodynamically stable, and was weaned off of all mechanical, circulatory, and ventilatory support over the next $24 \mathrm{~h}$. Echocardiogram performed immediately postmyocardial infarction showed a left ventricular ejection fraction (LVEF) of $25 \%$ with abnormal anterior-wall motion; this recovered to normal LVEF on repeat echocardiogram prior to discharge. The patient was discharged to home in ambulatory state from her index hospitalization.

\section{Discussion}

Our report presents a case of a patient with favorable recovery from early ECMO initiation after an IHCA.

In recent years, there has been a growing interest in utilizing E-CPR to improve both short- and long-term morbidity and mortality in patients postcardiac arrest. Review of the current medical literature yields mixed results on the use of E-CPR. ${ }^{[3-6]}$ However, a common variable that seems to determine success is the amount of delay in the initiation of ECMO postarrest. ${ }^{[3-5]}$ One trial of 57 patients

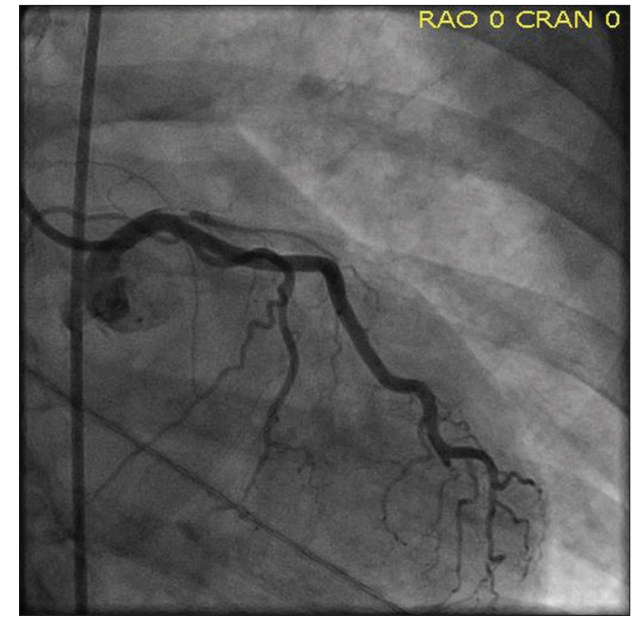

Figure 2: Angiograph showing occlusion of the ostial left anterior descending coronary artery

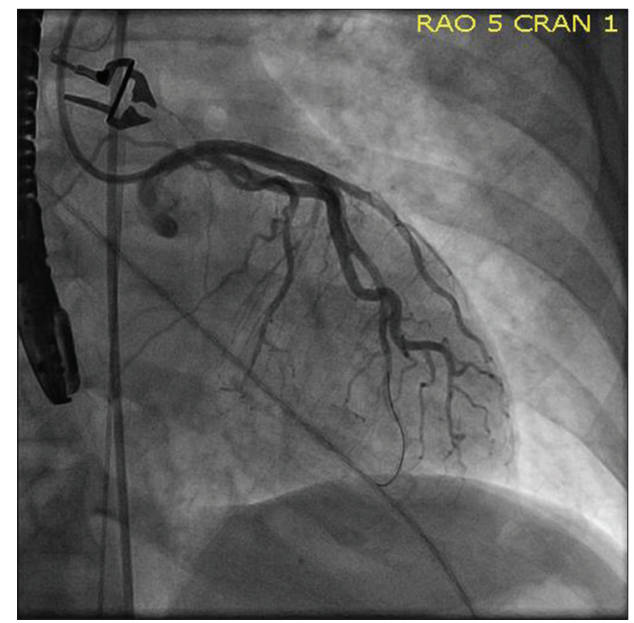

Figure 3: Postpercutaneous interventional image of the left anterior descending coronary artery

had a survival rate-defined as alive at discharge-of $31.6 \%$ with the average onset to ECMO at $39.2 \mathrm{~min} \pm 13.7$ in 
the survivor cohort compared to nonsurvivors' average of $51.5 \pm 11.3(P=0.004) .^{[3]}$ The CHEER trial achieved a $46 \%$ overall survival (defined as good neurological function at discharge) with the mean time to onset of ECMO of $40 \mathrm{~min}$ among the survivors as compared to $78 \mathrm{~min}$ among the nonsurvivors $(P=0.02) \cdot{ }^{[4]}$ On the other hand, an meta-analysis involving 1282 patients with OHCA and treated with E-CPR yielded $26.7 \pm 1.4 \%$ survival to discharge among all-comers. Furthermore, only $8.5 \%$ of the total patient population was alive at 1 month. ${ }^{[5]}$ However, the average time to ECMO initiation was $52 \mathrm{~min}$.

Prompt implementation of E-CPR in refractory cardiopulmonary arrest may be the next breakthrough required to improve outcomes post IHCA and OHCA. In our case, VA-ECMO was initiated to stabilize the patient and help sustain ROSC while the patient was treated for a reversible etiology of her V-Fib arrest. Time to ECMO support is expected to be shortened as more hospitals establish designated multidisciplinary cardiogenic shock teams. While larger randomized controlled trials are required before generalization of $\mathrm{E}-\mathrm{CPR}$, it certainly promises to be a lifesaving intervention in the prompt clinical setting.

\section{Conclusion}

In patients presenting with known and reversible etiologies of cardiopulmonary arrest, early initiation of ECMO in the peri-CPR phase may improve survival outcomes, especially at major academic centers with established multidisciplinary cardiogenic shock.

\section{Declaration of patient consent}

The authors certify that they have obtained all appropriate patient consent forms. In the form the patient(s) has/have given his/her/their consent for his/her/their images and other clinical information to be reported in the journal. The patients understand that their names and initials will not be published and due efforts will be made to conceal their identity, but anonymity cannot be guaranteed.

\section{Financial support and sponsorship}

Nil.

\section{Conflicts of interest}

There are no conflicts of interest.

\section{REFERENCES}

1. Merchant RM, Yang L, Becker LB, Berg RA, Nadkarni V, Nichol G, et al. Incidence of treated cardiac arrest in hospitalized patients in the United States. Crit Care Med 2011;39:2401-6.

2. Ofoma UR, Basnet S, Berger A, Kirchner HL, Girotra S; American Heart Association Get With the Guidelines - Resuscitation Investigators. Trends in survival after in-hospital cardiac arrest during nights and weekends. J Am Coll Cardiol 2018;71:402-11.

3. Chen YS, Chao A, Yu HY, Ko WJ, Wu IH, Chen RJ, et al. Analysis and results of prolonged resuscitation in cardiac arrest patients rescued by extracorporeal membrane oxygenation. J Am Coll Cardiol 2003;41:197-203

4. Stub D, Bernard S, Pellegrino V, Smith K, Walker T, Sheldrake J, et al. Refractory cardiac arrest treated with mechanical CPR, hypothermia, ECMO and early reperfusion (the CHEER trial). Resuscitation 2015;86:88-94.

5. Morimura N, Sakamoto T, Nagao K, Asai Y, Yokota H, Tahara Y, et al. Extracorporeal cardiopulmonary resuscitation for out-of-hospital cardiac arrest: A review of the Japanese literature. Resuscitation 2011;82:10-4.

6. Chen YS, Lin JW, Yu HY, Ko WJ, Jerng JS, Chang WT, et al. Cardiopulmonary resuscitation with assisted extracorporeal life-support versus conventional cardiopulmonary resuscitation in adults with in-hospital cardiac arrest: An observational study and propensity analysis. Lancet 2008;372:554-61. 